\title{
Arranging the high-confidence microRNA binding regions into web tool for analyzing variants in diagnostic next generation sequencing
}

\author{
O. Plotnikova ${ }^{1 *}$, M. Skoblov ${ }^{1,2}$ \\ ${ }^{1}$ Moscow Institute of Physics and Technology, Moscow, Russia \\ ${ }^{2}$ Research Centre for Medical Genetics, Moscow, Russia \\ *e-mail: Plotnikova@phystech.edu
}

Key words: Regulation of gene expression, microRNA-mRNA interactions, microRNA, miRNA, nucleotide variants

Motivation and Aim: It is well-known that microRNA plays a key role in the gene expression regulation. Today it is known more than 2'500 human microRNAs, while a majority of microRNA-mRNA interactions remain unidentified. A common method for determining the microRNA-mRNA interactions is the use of prediction programs that show a small intersection of the results and are poorly consistent with known experimental data [1]. Therefore, using experimentally obtained data of the microRNA binding regions should be the most promising approach in order to identify new molecular mechanisms for the etiopathogenesis of hereditary diseases.

Methods and Algorithms: We collected results of 79 AGO2-CLIP-seq data (18 PARCLIP and 61 HITS-CLIP) from 9 different cell lines. We also took data from two modified CLIP-seq studies (CLASH, CLEAR-CLIP) [2,3] that straightforwardly detect microRNA-mRNA pairs as chimeric reads. We developed the algorithm for analyzing the microRNA binding regions.

Results: Totally, we revealed 156 thousand microRNA binding regions and formed a subset of 46.8 thousand high-confidence regions that were identified in at least two different experiments. The analysis of high-confidence microRNA binding sites revealed tissue-specific interactions for two predominate cells: HEK293 and Huh7.5. On the other hand, we obtained a group of "house-keeping" microRNA binding regions that were identified in predominant cells. The identified high-confidence microRNA binding regions have been formed in web-tool. This tool analyzing nucleotide variants from patients with an inherited disease which could be caused by breaking in the microRNAmRNA interactions.

Conclusion: We identified the subset of 46.8 thousand high-confident human microRNA binding regions that were arranged in the tool (available online: http://score.generesearch. $\mathrm{ru} /$ services/mirna/). Hence, it will be a valuable resource that should provide additional insights into the identification new molecular mechanisms of hereditary diseases caused by breaking in the microRNA-mRNA interactions.

\section{References}

1. Plotnikova O., Skoblov M. (2018) Efficiency of the miRNA-mRNA interaction prediction programs. Molecular Biology. 52(2) (in press).

2. Helwak A. et al. (2013) Mapping the human miRNA interactome by CLASH reveals frequent noncanonical binding. Cell. 153(3):654-665.

3. Moore M.J. et al. (2015) miRNA-target chimeras reveal miRNA 3 '-end pairing as a major determinant of Argonaute target specificity. Nature Communications. 6:8864. 